\title{
GRAVIDEZ PÓS-TRANSPLANTE RENAL: ANÁLISE CRÍTICA DOS ASPECTOS FISIOPATOLÓGICOS
}

\author{
Pregnancy after renal transplantation: critical analysis of the physiopathology aspects
}

\author{
Alexandre Henrique Fiquerôa Moura ${ }^{1}$, Elson Roberto Ribeiro Faria², Vilber Antônio de Oliveira Bello4, Maria Cristina S. Lobo³, Dídimo \\ Carvalho Teles', Pedro Joely de Aquino e Moura'.
}

\section{RESUMO}

A maioria dos dados relatados sobre gravidez em receptores de órgãos sólidos refere-se a transplantadas renais. Este fato está associado à reabilitação e à melhoria da fertilidade em praticamente todas as pacientes, por meio do restabelecimento da função endócrina e menstrual que, em geral, ocorre entre um e 30 meses após o transplante. A incidência de rejeição em transplantados renais durante a gravidez é de $9 \%$, dado este estatisticamente não significante em relação à população de receptoras não-grávidas. Os episódios de rejeição, quando presentes, influenciam seriamente o resultado da gestação, devido ao comprometimento da função renal. Nas receptoras de transplante renal, o índice de prematuridade oscila entre 45 e $65 \%$, independente do esquema imunossupressor adotado ou do intervalo de tempo entre o transplante e a gestação. Dados do National Transplantation Pregnancy Registry (N.T.P.R), a partir de um estudo de caso - controle de gravidez após transplante renal, não revelou qualquer influência estatisticamente significativa a respeito da gestação sobre a função do enxerto. Concluindo, o transplante renal reabilita a paciente portadora de insuficiência renal crônica terminal para a gestação. Por outro lado, a gestação não representa fator limitante para a manutenção da função do enxerto no pós-transplante renal.

Descritores: gravidez, transplante renal, fisiopatologia, enxerto, insuficiência renal terminal.

Trabalho realizado na Unidade de Transplante Renal do Hospital de Base do Distrito Federal - Brasília - Distrito Federal - Brasil.

1. Especialistas em urologia e titulares da Sociedade Brasileira de Urologia

2. Mestre em Urologia e Doutor em Ciências pela Faculdade de Medicina da Universidade de São Paulo;

3. Especialista em nefrologia e titular da Sociedade Brasileira de Nefrologia

4. Mestre em Nefrologia e Titular da Sociedade Brasileira de Nefrologia

Endereço para correspondência: Elson Roberto Ribeiro Faria - SHLS 716, Centro Clínico Sul, Ala Central, Sala C-7 - CEP: 70.0833-050 - Brasília - D.F. - Telefone: (61) 3345-4200 Fax: (61)3346-7612

E-mail: e_faria@terra.com.br_ultradf@terra.com.br

Recebido em: 19/10/04
Aceito em: 29/04/05

\section{INTRODUÇÃO}

O transplante renal representa a melhor forma de tratamento para os pacientes portadores de insuficiência renal crônica terminal. Proporciona, entre outras coisas, significativa melhoria da qualidade de vida, prolongamento da sobrevida e retorno à capacidade fértil do indivíduo.

Com os recentes avanços da terapia imunossupressora, tem-se obtido progressiva melhoria de sobrevida do enxerto e, com isto, a possibilidade do retorno pleno às atividades sociais, profissionais $\mathrm{e}$ afetivas. A gestação constitui um atributo, uma fenomenal conquista da mulher, e não poderia ser diferente para aquela que se sente plenamente realizada e bem sucedida com o seu transplante renal.

O primeiro relato de gravidez pós-transplante renal ocorreu em 1958. A doadora renal foi a irmã gêmea idêntica e, desde então, mais de 2.000 gestações foram informadas. ${ }^{1}$

A maioria dos dados relatados sobre gravidez em receptores de órgãos sólidos refere-se a transplantadas renais. Este fato está associado à reabilitação e à melhoria da fertilidade em praticamente todas as pacientes por meio do restabelecimento da função endócrina e menstrual que, em geral, ocorre entre um e 30 meses após o transplante. ${ }^{2}$

Alguns autores consideram a gravidez próspera como a melhor forma possível de provar que houve reabilitação da insuficiência renal crônica. ${ }^{4,5,6,7}$ Em 1993, alguns pesquisadores ${ }^{3}$ mencionaram que a taxa de concepção, na faixa etária entre 15 e 45 anos, é de uma em cada 50 receptoras. Referiram, também, que a possibilidade de 
evolução favorável da gestação é de 55 a 90\% em receptoras com a função renal estável. ${ }^{3}$

A possibilidade de danos ao enxerto, proporcionada por alterações decorrentes das mudanças orgânicas causadas pela gravidez, assim como o maior risco de complicações no desenvolvimento da gestação, são preocupações constantes para os profissionais que lidam com pacientes transplantados. Dentre as mais importantes complicações gestacionais pós-transplante estão a perda da função do enxerto, a hipertensão arterial, a recidiva da doença primária e os efeitos tóxicos das drogas imunossupressoras. ${ }^{3}$

O papel dos agentes imunossupressores durante a gestação também merece algumas considerações, principalmente, no que concerne ao alto índice de prematuridade. A ruptura prematura de membranas decorrente do uso de corticóides representa a principal etiologia do parto prematuro. ${ }^{4}$

O presente estudo tem por objetivo promover uma revisão bibliográfica sobre a gravidez em receptoras de transplante renal, com ênfase às principais complicações ocorridas durante a gestação e o impacto deste estado sobre a função do enxerto.

\section{O MECANISMO ADAPTATIVO DA GRAVIDEZ}

\subsection{Alterações fisiológicas da função renal durante a gravidez}

A gravidez é responsável por importantes modificações no organismo materno, dentre as quais observam-se substanciais alterações fisiológicas da função renal.

$\mathrm{Na}$ mulher normal e normotensa, a gravidez proporciona um significativo aumento do ritmo de filtração glomerular (RFG). Tal fenômeno ocorre nas primeiras semanas de gestação, atingindo níveis máximos ao redor da $15^{\mathrm{a}}$ semana e persistindo até a $36^{\mathrm{a}}$ semana. Ao final da gestação, ocorre progressivo retorno do RFG a níveis pré-gestacionais. O fluxo plasmático renal (FPR) elevase também, concomitantemente ao RFG, e diminui próximo ao parto. ${ }^{5,6,7}$

Embora estas alterações hemodinâmicas renais estejam bem estabelecidas, é de fundamental importância considerar o caráter individual, bem como a metodologia utilizada para estimar os parâmetros da função renal, haja vista que durante a gravidez ocorrem modificações fisiológicas renais e metabólicas de substâncias normalmente utilizadas para aferir o RFG e o FPR. Adicionalmente, a correção desses parâmetros pela superfície corporal é também um fator importante na avaliação da função renal durante a gestação. Na maioria das vezes, observa-se que a filtração glomerular aumenta $50 \%$ ao longo da gravidez. Por sua vez, o fluxo plasmático renal atinge valores entre 60 e $80 \%$ acima do nível pré-gestacional, acompanhado por significativa redução do mesmo por volta do terceiro trimestre, e normalização do fluxo à época do puerpério. ${ }^{6,7}$

Por meio do desenvolvimento de modelos experimentais, usando técnicas de micropunção, os determinantes da filtração glomerular foram analisados e concluiu-se que o seu incremento em néfrons corticais de cobaias deve-se à diminuição da resistência em ambas as arteríolas pré e pós-glomerulares. ${ }^{8}$

O aumento do fluxo plasmático glomerular é proporcional entre ambas as arteríolas, não modificando a pressão intrarteriolar. Porém, não foi esclarecido se a vasodilatação está meramente relacionada ao tempo ou é precipitada pela gravidez..$^{8,9,10}$
Em 1989, pesquisadores ${ }^{11}$ mencionaram que o coeficiente de ultrafiltração glomerular também não é alterado durante a gestação. Assim, o principal mecanismo determinante do aumento da filtração glomerular é a elevação do fluxo plasmático em conseqüência da vasodilatação intra-renal.

A manutenção da pressão intra-glomerular durante a gravidez normal é extremamente necessária, principalmente, pelo fato de que a vasodilatação intra-renal crônica pode comprometer a estrutura do glomérulo. Esse dano glomerular é agravado pela hipertensão arterial, na qual a vasodilatação é preponderante na arteríola préglomerular. Tal achado é observado nos pacientes portadores de nefropatia diabética e nos casos em que ocorre a redução do número de néfrons funcionantes. Nessa última condição, a vasodilatação intra-renal é acompanhada por hipertensão glomerular e conseqüente desenvolvimento de esclerose glomerular. ${ }^{8,9,10}$

\subsection{Mediadores da vasodilatação intra-renal}

O sistema endócrino materno passa por significativas alterações hormonais que podem proporcionar modificações hemodinâmicas. Várias substâncias e alguns hormônios têm sido indicados como possíveis mediadores dessas alterações renais induzidas pela gravidez. Dentre os prováveis mediadores responsáveis pela vasodilatação intra-renal durante o período gestacional, as prostaglandinas, a prolactina, a progesterona e o óxido nítrico (NO) constituem seus principais representantes. ${ }^{12,13,14,15,16}$

\section{Prostaglandinas}

As prostaglandinas e, em particular, as de ação vasodilatadoras, parecem exercer algum papel no mecanismo de vasodilatação intra-renal.

A excreção urinária dessas substâncias ou de seus metabólitos encontra-se elevada durante a gestação, tanto em humanos como em animais de experimentação. Entretanto, vários estudos empregando cobaias em diferentes condições experimentais mostraram que a administração de inibidores das prostaglandinas não foi capaz de reverter o aumento da depuração de creatinina gestacional. ${ }^{17,18}$

Adicionalmente, a biossíntese de prostaglandinas pelos glomérulos não é diferente entre ratas virgens e grávidas. Essas experiências indicaram, portanto, que as prostaglandinas não devem ter participação importante no mecanismo de vasodilatação intrarenal induzido pela gestação, pelo menos quando analisadas

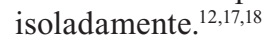

\section{Prolactina e Progesterona}

A participação destes hormônios durante a gravidez ainda permanece sob investigação experimental e com resultados iniciais demonstrando controvérsia sobre a hemodinâmica renal ${ }^{13,14}$. Alguns estudos mostraram que a hiperprolactinemia crônica pode induzir aumentos significativos da filtração e do fluxo renal ${ }^{19}$, enquanto outros autores não conseguiram repetir os mesmos resultados.

\section{Óxido Nítrico (NO)}

$\mathrm{O}$ óxido nítrico (NO), fator relaxante derivado do endotélio, aparentemente está implicado com a vasodilatação intra-renal, em especial com a hiperfiltração desenvolvida durante a gravidez. Isto se deve, particularmente, ao fato da excreção urinária do NO e seus metabólitos encontrar-se substancialmente elevada neste período, 
tanto em seres humanos como em animais de experimentação, segundo a literatura. ${ }^{16}$

A inibição aguda ou crônica do NO é capaz de reverter a vasodilatação e a hiperfiltração glomerular em cobaias. ${ }^{20}$ Pesquisadores demonstraram que a inibição crônica resultou em hipertensão e redução da função renal com dano ao endotélio, à semelhança da pré-eclâmpsia. ${ }^{16,20}$

A manutenção da vasodilatação intra-renal parece ser uma condição fundamental para a evolução normal da gravidez, havendo, portanto, necessidade de mecanismos compensatórios que possam ser recrutados na vigência de falha de um sistema primário. Recentemente foi demonstrado que a inibição crônica do $\mathrm{NO}$ em ratas não foi capaz de abolir totalmente a vasodilatação sistêmica e renal da gravidez. Porém, quando a inibição do NO foi feita conjuntamente com o bloqueio das prostaglandinas, tanto a vasodilatação como a hiperfiltração glomerular foram interrompidas. Estes experimentos confirmaram que o NO tem papel fundamental na vasodilatação induzida pela gravidez e sugeriram que as prostaglandinas, embora não produzam efeito, quando analisadas isoladamente, podem ter um papel compensatório durante a inibição crônica do NO., ${ }^{716,20}$

\subsection{Mecanismos de regulação dos eletrólitos}

Durante, praticamente, todo o período gestacional, ocorre aumento da reabsorção de sal e água. Essa elevação da reabsorção tubular de fluído surge como resultado do aumento da filtração glomerular, evitando assim, uma depleção materna do volume extracelular. No entanto, ainda não se sabe claramente quais os mecanismos envolvidos e nem os locais ao longo do néfron responsáveis por tal reabsorção. O túbulo proximal, a alça ascendente espessa de Henle e o ducto coletor parecem estar envolvidos, principalmente, na reabsorção de sódio, cloro e água. ${ }^{7}$ A excreção renal de outros solutos, incluindo ácido úrico, cálcio e magnésio, aumenta durante a gravidez normal. Ao contrário, a excreção de potássio, glicose e uréia, diminui, porém, por mecanismos ainda não esclarecidos. ${ }^{7}$

\section{REJEIÇÃO APÓS O TRANSPLANTE RENAL}

A rejeição permanece como o problema crítico no pós-transplante renal. Os mecanismos imunológicos responsáveis pela rejeição do enxerto são heterogêneos e envolvem a ativação imunológica humoral e celular. ${ }^{20,21,22}$

Há referência de 1992, afirmando que a gravidez pode ser considerada como um transplante intra-uterino próspero ${ }^{21}$. O desenvolvimento de um feto no corpo da mãe, apesar dos antígenos de histocompatibilidade mutuamente incompatíveis, representa um fenômeno desafiador contínuo para a imunologia. ${ }^{21}$

A incidência de rejeição em transplantados renais durante a gravidez é de $9 \%$, dado este estatisticamente não significativo em relação à população de receptoras não-gravídicas. ${ }^{22}$ Freqüentemente, a incidência de rejeição do enxerto em gestante é até menor. Segundo alguns autores ${ }^{3}$, os episódios de rejeição, quando presentes, prejudicam seriamente o resultado da gestação, devido ao comprometimento da função renal, o que pode redundar em retorno à diálise até em $18 \%$ das pacientes. De acordo com os dados do National Transplantation Pregnancy Registry (N.T.P.R), em que pesquisadores ${ }^{22}$ avaliaram receptoras renais com intervalo entre a gravidez e o transplante superior a 5 anos e função renal preservada, não mostraram episódios de rejeição, o que demonstra a importância da estabilidade da função renal como fator preditivo.

Outra condição importante no controle da rejeição seria a própria gravidez, a qual exerceria um papel imunoregulatório, caracterizando, assim, um período imunológico privilegiado. ${ }^{22}$

Embora a introdução de novos agentes imunossupressores possa viabilizar um melhor controle sobre a rejeição aguda e conseqüente aumento da taxa de sucesso dos transplantes de órgãos, a sobrevida do enxerto em longo prazo pode ser limitada pelo surgimento da rejeição crônica. ${ }^{23}$ Portanto, a prevenção da rejeição continua sendo a primeira meta a ser atingida em transplante de órgãos sólidos.

\section{IMUNIDADE ESPECÍFICA DURANTE A GESTAÇÃO}

O mecanismo de tolerância imune específica durante a gestação ainda é pobremente compreendido. O fenômeno é caracterizado por eventos imunológicos humorais e celulares que induzem tolerância para os antígenos paternos e, dessa forma, podem transmitir a informação necessária à promoção de tolerância específica ou não específica em transplantes de órgãos. Existe um forte processo ativo imunoregulatório natural na gravidez que parece estar relacionado à atividade da célula supressora. Por meio de anticorpos bloqueadores específicos, a célula supressora torna-se responsável pelo desenvolvimento de não - responsividade alogênica específica materna. ${ }^{24}$

Em transplante de órgão, a sobrevivência do enxerto em longo prazo sem o uso de drogas imunossupressoras é praticamente impossível. Contudo, alguns casos têm sido relatados ${ }^{25}$. O regime imunossupressor normal deve ser continuado durante a gravidez, proporcionando uma sobrevida estável do enxerto, em longo prazo. ${ }^{26}$

A deteriorização da função renal acontece em apenas 15\% das grávidas que foram submetidas ao transplante de rim $^{27}$. Portanto, os processos imunoregulatórios naturais ativos induzidos pela gravidez podem, também, ter representado um papel permissivo no desenvolvimento da não-resposta imunológica específica para o enxerto renal nestas pacientes. ${ }^{27}$

\section{IMUNOSSUPRESSÃO DURANTE A GRAVIDEZ}

\subsection{Esquema clássico}

A necessidade de estabelecer os danos produzidos pelos imunossupressores durante a gravidez fez com que vários autores tentassem correlacionar o mecanismo de ação destes agentes com as principais complicações ocorridas na gravidez.

A azatioprina e a prednisona, por terem sido utilizadas no esquema clássico inicial, foram estudadas em detalhes e suas complicações já estão estabelecidas. A ruptura da membrana amniótica pelo uso crônico de corticóides ocorre em $40 \%$ dos casos e representa uma das principais causas de prematuridade, presente em cerca de 59\% das pacientes. ${ }^{4}$

A correlação entre malformações congênitas e o uso desses agentes ainda não foi totalmente determinada, muito embora doses elevadas de azatioprina em animais de laboratório tenham produzido efeito teratogênico ${ }^{27}$. Anormalidades cromossômicas também têm sido descritas em linfócitos de crianças, filhas de transplantadas renais. No entanto, tais alterações desapareceram com o tempo. De qualquer modo, existe a possibilidade de surgimento de anomalias congênitas em gerações futuras, segundo a publicação de Hadi em 1986. ${ }^{28}$ 


\subsection{Ciclosporina}

A partir de 1983 a ciclosporina (CsA) começou a ser utilizada e, desde então, vêm surgindo dúvidas sobre a influência desse agente na evolução da gravidez. Em vários centros transplantadores foram iniciados protocolos prospectivos com o objetivo de identificar possíveis problemas.

Há referência de que a função renal em longo prazo, assim como os entraves relacionados à gestação ${ }^{29}$, mostraram-se com resultados desfavoráveis em receptores sob o uso de ciclosporina. Os autores constataram complicações obstétricas, tais como o baixo peso, o crescimento uterino retardado e a prematuridade. No entanto, tais resultados foram pouco significantes estatisticamente em relação ao que foi observado nas pacientes que não utilizavam CsA em sua terapia. ${ }^{29}$

Por meio de dados catalogados pelo N.T.P.R., foram avaliadas as possíveis influências deste agente por meio do seguimento de pacientes mantidos com CsA antes, durante e depois da gravidez, comparando-os com o grupo que utilizava o esquema convencional. As pacientes em uso de imunossupressão com azatioprina e prednisona aparentemente completaram a gestação com melhor função renal em comparação àquelas que faziam uso de ciclosporina. Esta diferença foi estatisticamente significativa em relação a creatinina sérica. $\mathrm{O}$ estudo em questão usou grupos semelhantes, emparelhados, selecionados e com sobrevida extremamente satisfatória ${ }^{22}$. Foi constatada uma acelerada deteriorização da função renal, aferida pelo nível sérico de creatinina, após o início da gravidez em comparação ao período anterior. Além disso, a sobrevida do enxerto em longo prazo mostrou-se significativamente pior em pacientes grávidas se comparadas àquelas do grupo controle, que tiveram taxa de sobrevida do enxerto de $100 \% \mathrm{em}$ 10 anos. Tais achados constituíram os primeiros estudos de longo prazo da função renal após a gravidez ${ }^{22}$. Porém, esses efeitos são difíceis de ser determinado, já que a deteriorização da função renal ocorre com certa freqüência em transplante de órgãos. ${ }^{30}$

A ciclosporina ultrapassa a barreira placentária e seus metabólitos podem ser encontrados no sangue fetal, no cordão umbilical e no líquido amniótico em 10 a $50 \%$ dos casos. ${ }^{31}$ Segundo os autores, a possibilidade de um efeito citotóxico direto existe, porém, até o momento, nenhuma malformação fetal foi comprovadamente relacionada a ciclosporina.

Os níveis plasmáticos da CsA reduzem durante a gestação. Isto se deve às propriedades farmacocinéticas dessas drogas de ligar-se a eritrócitos e lipoproteínas, as quais encontra-se com taxas reduzidas após o $2^{\circ}$ trimestre. A partir deste momento ocorre aumento do volume plasmático. Tais fatos merecem atenção especial, uma vez que, durante a gravidez, tanto a rejeição quanto a nefrotoxicidade podem ocorrer. Segundo Thomas et al. ${ }^{29}$ a explicação para a ausência de rejeição na vigência de níveis baixos de CsA pode ser dada por dois motivos: o primeiro seria resultante da supressão auto-imune causada pelo próprio estado de gravidez; o segundo, resultaria de uma redução da propriedade terapêutica durante a gravidez, sem razão aparente.

A nefrotoxicidade deste agente pode interferir no fluxo plasmático renal, por meio de alterações na síntese de prostanóides, prejudicando, também, a circulação útero-placentária. ${ }^{29}$

\section{FATORES DE RISCO}

Em revisão à literatura, cuja análise envolveu 2.309 gestações em
1.593 receptoras, foi constatado que em $40 \%$ dos casos não houve progressão gestacional além do $1^{\circ}$ trimestre, como conseqüência de abortamento espontâneo ou terapêutico. Entre as gestações remanescentes, acima de $90 \%$ resultaram em nascidos vivos. ${ }^{27}$ Tais estudos evidenciaram, também, que essas gestações, consideradas de alta morbidade, estão associadas a vários fatores de risco tanto para a mãe como para o feto. No primeiro caso, pode levar à piora da função renal e à hipertensão arterial que, segundo a maior parte dos relatos, representa o principal fator de morbidade. ${ }^{27}$ A hipertensão arterial acontece em $26 \%$ das gestantes. ${ }^{26}$ Para o feto, os fatores de risco mais apontados são a prematuridade, o baixo peso e o crescimento uterino retardado, para os quais o esquema de imunossupressão parece representar o principal agente etiológico ${ }^{26}$.

\subsection{Prematuridade}

A prematuridade é a principal causa de mortalidade perinatal e um dos grandes desafios para os responsáveis pela assistência obstétrica. Trata-se de um problema não apenas médico, mas, sobretudo, de uma questão social e humana. Aproximadamente $45 \%$ dos casos decorrem de partos prematuros com membranas íntegras, das quais $30 \%$ por ruptura de membranas amnióticas e $25 \%$ deles induzidos como recurso terapêutico para as complicações maternas ou fetais. ${ }^{32}$

A incidência da prematuridade em grávidas não-transplantadas é de difícil avaliação devido à influência de vários fatores, tais como a densidade populacional e a qualidade de assistência médica loco-regional. No entanto, a literatura mostra que, em países desenvolvidos, a taxa de prematuridade em geral é de 8 a $10 \%$ e em países em desenvolvimento varia de 5 a $15 \%{ }^{4}$

Nas receptoras de transplante renal, o índice de prematuridade oscila entre 45 e $65 \%$, independente do esquema imunossupressor adotado ou do intervalo de tempo entre o transplante e a gestação. ${ }^{33}$

A explicação para esta elevada incidência decorre de vários fatores, dentre eles: a ruptura prematura de membrana amniótica, que ocorre em 20 a $40 \%$ dos casos e é atribuída ao uso crônico de corticóides $^{2}$; a alta incidência de infecções do trato urinário e as intervenções obstétricas, usualmente empregadas em pacientes com hipertensão arterial não-controlada. ${ }^{4}$

\subsection{Intervalo de tempo entre o transplante e a gravidez}

O intervalo de tempo transcorrido entre o transplante e o início da gravidez pode ser considerado um dos fatores que influenciam a evolução favorável da gestação. Estudo realizado em 1995 mostrou que intervalo de tempo inferior a dois anos está associado a menor incidência de complicações (prematuridade, baixo peso e perda do enxerto) quando comparados a receptores com intervalo superior a dois anos. Este fato está diretamente relacionado à estabilidade da concentração dos imunossupressores e da função do enxerto nestas pacientes. ${ }^{2}$

\subsection{Abortamento}

A interrupção espontânea da gravidez, em mulheres em geral e sem antecedentes de alteração da função renal, ocorre em cerca de $16 \%$ dos casos. ${ }^{30} \mathrm{Em}$ receptoras de transplante renal, esta incidência apresenta-se mais elevada, atingindo $23 \%$ das grávidas. ${ }^{4}$ Em muitos casos, o fenômeno deve-se à utilização de medicações 
que dificultam a manutenção da gestação. Um exemplo é o uso de anticoagulantes orais, medicações que podem fazer parte do arsenal terapêutico utilizado nestas pacientes. ${ }^{4}$

Outro fator relacionado à interrupção espontânea da gravidez é o abortamento terapêutico, bastante empregado nos casos em que o controle da hipertensão arterial não é alcançado. Porém, este tipo de medida encontra-se em franco declínio nos últimos anos, demonstrando uma melhora das condutas frente a tais pacientes. A participação dos centros transplantadores, seja por meio de esclarecimentos ou de um controle rigoroso dessas receptoras, também tem contribuído de forma significativa para a redução deste tipo de procedimento. ${ }^{4}$

O esquema imunossupressor e o intervalo de tempo entre o transplante e a gravidez foram, inicialmente, considerados fatores de risco relacionados ao abortamento. Porém, recentemente, estudo retrospectivo observacional realizado por meio do N.T.P.R., em que se avaliaram receptores renais com intervalo de tempo superior a cinco anos e tratados com ciclosporina, mostrou resultados similares àqueles em receptores com intervalos pequenos e em uso de azatioprina e prednisona. No entanto, os grupos comparados apresentavam enxerto com função renal satisfatória antes da gravidez, sugerindo que a presença de função renal adequada (creatinina $<1,3 \mathrm{mg} / \mathrm{dl}$ ) contribui de forma satisfatória para a progressão gestacional. ${ }^{22}$

Abortamentos habituais podem ser causados pelo fracasso dos anticorpos bloqueadores específicos em mascarar antígenos envolvidos em rejeição fetal. Assim, tais abortamentos são considerados como um tipo de rejeição contra antígenos fetais ${ }^{34}$.

Pesquisas realizadas em $1989^{35,36}$ referiram que abortamentos habituais seriam prevenidos por meio de transfusão de leucócitos do marido. De acordo com esta observação, transfusões de sangue teriam um efeito benéfico na taxa de sobrevivência do enxerto, o que sugeriu que mecanismos imunológicos semelhantes pudessem acontecer no período gestacional. Alguns estudos mostraram que uma maior disparidade genética materna e paterna contribui para o sucesso da gravidez, sendo este um possível fator responsável pelo processo imunoregulatório ativo e protetor contra a destruição imunológica do embrião. ${ }^{37}$

\subsection{Hipertensão arterial}

A hipertensão arterial representa uma das mais freqüentes causas de interrupção da gestação no primeiro trimestre em transplantadas renais. Cerca de $30 \%$ das pacientes hipertensas na pré-gestação evoluem com pré-eclâmpsia. Dessa forma, o controle rigoroso dos níveis pressóricos durante a gestação é fundamental para que a gravidez evolua de forma satisfatória. ${ }^{26}$

A hipertensão severa em receptores renais constitui contraindicação absoluta para a gravidez. No entanto, formas leves de hipertensão arterial, controladas com medicações, apresentam-se com índices de complicações gestacionais semelhantes às pacientes receptoras renais normotensas ${ }^{26}$. Segundo os autores, a incidência de efeitos adversos durante a gravidez é maior que a esperada na população com níveis semelhantes de hipertensão essencial, indicando que os transplantados renais têm mais susceptibilidade aos efeitos sistêmicos da hipertensão. ${ }^{26}$

A etiologia da hipertensão também representa um importante fator prognóstico. Tem-se postulado que lesões microvasculares associadas à hipertensão crônica poderiam, teoricamente, alterar o mecanismo adaptativo da gravidez, comprometendo-se, assim, a circulação útero-placentária. Acredita-se, também, que a presença dos rins primitivos, em muitos casos responsáveis por alterações na regulação do sistema renina-angiotensina, poderia contribuir para a permanência da hipertensão. No entanto, o controle rigoroso dos níveis pressóricos até, aproximadamente, a $28^{\mathrm{a}}$ semana, está associado à evolução satisfatória da gestação. ${ }^{26}$

Incidência aumentada de gravidez induzindo à doença hipertensiva em 26 a $30 \%$ das pacientes transplantadas renais, foi demonstrada em 1982.38 A diferenciação entre hipertensão e doença hipertensiva exclusiva da gravidez, às vezes, pode ser difícil, uma vez que os níveis pressóricos elevados, a proteinúria e o edema podem acontecer durante essas gestações sem a presença de doença hipertensiva exclusiva da gestante. $\mathrm{Na}$ gravidez múltipla, isto constitui um fator de risco para o precoce aparecimento de préeclâmpsia. A maneira de tratar tal complicação deve diferir em relação a pacientes não-transplantadas. Em caso de pré-eclâmpsia severa, o regime terapêutico de escolha é a interrupção da gravidez, independentemente da idade gestacional. ${ }^{26}$

\section{GRAVIDEZ MÚLTIPLA PÓS-TRANSPLANTE RENAL}

$\mathrm{Na}$ atualidade, o número de mulheres submetidas ao transplante renal em idade reprodutiva é cada vez maior. Sendo assim, é esperado que o número de gestações em receptores renais aumente. Em 1999, estudiosos do assunto ${ }^{39}$ informaram que o número de gestações prósperas pós-transplante renal aumentou continuamente na Europa, paralelamente ao número crescente de mulheres em idade fértil.

Mulheres portadoras de insuficiência renal crônica terminal (I.R.C.T.) e em programa dialítico normalmente apresentam ciclos anovulatórios e infertilidade. A gravidez, nesses casos, acontece numa proporção de uma para cada 200 mulheres em idade fértil e, muito poucas vezes, resultam em nascimento a termo. ${ }^{40}$

A possibilidade de concepção em mulheres na idade reprodutiva depois do transplante renal eleva-se rapidamente e, portanto, é fundamental proceder à orientação dessas pacientes quanto aos cuidados e riscos de uma possível gravidez. Nos casos de mulheres em que, após a realização do transplante renal, a função endócrina seja restabelecida parcialmente ou exista algum fator que impossibilite a fecundação, a opção por técnicas de fertilização assistida, naquelas que objetivam a gravidez, pode ser utilizada. Nessa situação, as chances de uma gestação múltipla encontramse elevadas. Essas receptoras enquadrar-se-iam num grupo de alto risco, criando um dilema de administração, cujo resultado obstétrico inclui, além de complicações maternas, um efeito perinatal adverso na maioria dos casos. ${ }^{39}$

No que se refere à utilização de técnicas de reprodução assistida, algumas medidas podem ser usadas a fim de reduzir o índice de gravidez multifetal. Dentre elas, o uso cuidadoso de agentes de indução ovulatória e a transferência de um número limitado de embriões. ${ }^{39}$ Caso a gravidez multifetal ocorra, apesar dos cuidados adotados, a possibilidade de redução fetal deve ser discutida, uma vez que há relatos de bons resultados quando esta opção foi empregada. No entanto, a grande dificuldade em adotar tal medida é a pouca aceitação por parte dos pais, haja vista que a participação dos mesmos é fundamental para a adoção da referida conduta. ${ }^{39}$

A prematuridade entre pacientes transplantadas renais oscila entre 
45 e $65 \%$ dos casos. Em relação à gravidez multifetal, a incidência não está definida devido ao número reduzido de casos. Porém, acredita-se ser ainda maior. Portanto, a possibilidade de redução do número fetal torna-se uma proposta atraente com o objetivo de diminuir o risco desta complicação. ${ }^{33}$

Pesquisas publicadas em $1993^{41}$ relataram que o êxito da redução fetal em seis grandes centros foi de $100 \%$. Outros estudiosos ${ }^{39}$ observaram que a taxa de perda, quando não houve redução fetal, foi de 8 a $9 \%$ em gestação inferior a 24 semanas. Os benefícios da redução fetal, no caso de trigêmeos e gêmeo, superam a tentativa de manutenção da gravidez múltipla nessas pacientes. ${ }^{39}$

São limitados os dados encontrados na literatura com relação à conduta frente a essas pacientes transplantadas renais com gravidez múltipla. Há relatos de experiências bem sucedidas, nas quais foram tratadas duas pacientes com gravidez múltipla pós-transplante renal. Uma delas teve trigêmeos e a outra uma gravidez gemelar. Os gêmeos evoluíram com gestações de 38 semanas, sem complicações maternas ou fetais. ${ }^{39}$

Estudos científicos ${ }^{42}$ informaram resultados favoráveis em $6 \%$ dos casos de gêmeos numa série de 17 gestações. A Associação Européia de Diálise e Transplante Renal (E.D.T.A.) registrou 120 casos de gravidez bem sucedida, onde 3\% foram gêmeos e 1\% trigêmeos. ${ }^{43}$

Estudos clínicos demonstraram que a pré-eclâmpsia atinge 26 a $30 \%$ das pacientes transplantadas. ${ }^{9,38,44}$ Numa combinação de transplante renal e gravidez múltipla a incidência esperada de pré-eclâmpsia é ainda mais elevada, de acordo com os autores.

\section{GRAVIDEZ REPETIDA PÓS-TRANSPLANTE RENAL}

Em receptoras com a função renal estável, as alterações decorrentes de uma gravidez nem sempre comprometem a função do enxerto, fato este que encoraja e faz com que gestações repetidas sejam observadas em vários centros transplantadores ${ }^{22}$. O aconselhamento psicológico, a experiência de vida com a gestação anterior, assim como uma rigorosa avaliação médica, faz parte dos critérios empregados no controle destas pacientes. No entanto, alguns dados específicos sobre a análise da função renal nestes casos ainda são escassos.

Uma análise multicêntrica realizada por meio do N.T.P.R., durante o período de 1967 a 1990, evidenciou cerca de 820 gestações bem sucedidas em 718 receptoras. O resultado mostrou que apenas $2 \%$ destas pacientes retornaram à diálise e somente em um dos casos ocorreu perda do enxerto durante a gravidez; as demais perdas aconteceram entre dois e nove anos após a gestação. Este fato reforça a idéia de que as alterações provocadas pelo mecanismo adaptativo gestacional, que poderiam ser responsáveis pela deterioração da função renal, não são significativas em pacientes estáveis do ponto de vista funcional do enxerto. A presença de gestações repetidas não aumentou as chances de danos irreversíveis ao enxerto, desde que estes receptores se enquadrem no grupo de estáveis e estejam, portanto, aptos a desenvolver uma gravidez próspera. ${ }^{22}$

Experiências laboratoriais ${ }^{7}$ determinaram que múltiplas gestações em ratas normotensas não implicaram em piora da função renal em longo prazo, quando comparadas com ratas virgens da mesma idade. Por outro lado, outros estudiosos do tema ${ }^{45}$ demonstraram que ratas velhas submetidas a várias gestações apresentavam redução da filtração glomerular e do fluxo plasmático renal quando comparadas a ratas virgens da mesma idade. Este fato ocorria devido a uma elevação da resistência vascular renal, como resultado de um possível decréscimo na síntese de óxido nítrico. ${ }^{45}$ Esse conjunto de dados experimentais sugeriu que a função renal normal não é afetada na gravidez. No entanto, quando há associação de múltiplas gestações, a análise funcional revela um estado de vasoconstricção intra-renal no envelhecimento, provavelmente, decorrente de um déficit na síntese de NO, sugerindo possível lesão do endotélio vascular ?

Em relação às complicações presentes durante o desenvolvimento do feto (prematuridade, baixo peso e crescimento uterino retardado), parece não haver diferença entre a primeira gravidez e as subseqüentes. Tal fato é possível quando se considera que durante o intervalo entre as gestações não tenha havido alterações e que a hipertensão arterial, principal fator de co-morbidade, tenha sido controlada. Da mesma forma, as medidas antropométricas fetais, quando comparadas, também não mostraram diferenças. ${ }^{45}$

\section{IMPACTODA GRAVIDEZSOBRE AFUNÇÃORENALEM LONGO PRAZO EM PACIENTES TRANŞPLANTADAS}

A gravidez normal pode induzir alterações hemodinâmicas bem como modificações estruturais do rim, incluindo aquelas relacionadas à membrana do capilar glomerular, que em alguns casos, pode levar a albuminúria. Um estudo com mulheres caucasianas normais constatou a presença dessas alterações, as quais foram totalmente revertidas um ano após o parto. ${ }^{7}$ Segundo os autores, tal fato sugere que a gravidez pode ser caracterizada como um estado de vasodilatação intra-renal semi-crônico ou transitório, não constituindo qualquer motivo de dano renal.

A análise da função renal em longo prazo ainda constitui um exercício complicado, pois há dificuldade em estabelecer se a perda da função do enxerto é decorrente da gestação ou de fatores atribuídos ao tempo de transplante. ${ }^{30}$

Estudo tipo caso-controle de gravidez após transplante renal, a partir de dados do N.T.P.R. e baseado em relatórios multicêntricos ${ }^{22}$, não revelou qualquer influência estatisticamente significativa da gestação na função do enxerto. Contudo, o período de seguimento da função renal foi curto, entre 25 e 35 meses pós-parto ${ }^{22}$. Em 1992, pesquisadores ${ }^{33}$ apresentaram o resultado de uma análise, em um único centro, onde os níveis de creatinina plasmática, em 22 gestações, foram reduzidos progressivamente. Esse achado foi considerado como um indicador de ausência de efeito adverso da gravidez sobre a função do enxerto. ${ }^{33}$

\section{EXPERIÊNCIA DO HOSPITAL DE BASE DO DISTRITO FEDERAL}

No período de outubro de 1982 a maio de 1999, foram realizados 479 transplantes renais, sendo 135 do sexo feminino em idade fértil, no Hospital de Base do Distrito Federal. Sete pacientes $(5,1 \%)$ ficaram grávidas. Todas foram monitoradas em relação à função renal, por meio da dosagem de creatinina sérica antes, durante e após o período gestacional, e a aferição da pressão arterial, bem como em função do esquema de imunossupressão utilizado durante os respectivos intervalos.

Dos sete casos, cinco evoluíram com gestação a termo, sendo um parto normal e quatro cesarianas. Houve dois abortamentos. Todas as pacientes apresentavam função renal satisfatória antes da gravidez, a qual não sofreu alteração durante o período gestacional.

Não houve elevação da pressão arterial média durante o seguimento das pacientes no período gestacional. O esquema de imunossupressão variou conforme o grau de histocompatibilidade. 


\section{CONCLUSÃO}

O transplante renal reabilitou a paciente com IRCT para a gestação. Por outro lado, a gravidez pós-transplante renal não representou um fator limitante para a manutenção da função do enxerto, na experiência inicial da Unidade de Transplante Renal do Hospital de Base do Distrito Federal.

\section{ABSTRACT}

Most of the data on pregnancy in solid organ recipients is mentioned in kidney transplantation reports. This fact is correlated to the fertility rehabilitation and improvement in practically all patients due to the re-establishment of the endocrine and menstrual functions, which generally happens between 1 to 30 months after the transplant. The rejection incidence in these patients during pregnancy is of $9 \%$, which is statistically and non significantly related to a non pregnant population of receptors. When present, rejection episodes have an important influence on the result of the pregnant woman due to the compromising of the renal function. In kidney transplant patients, the prematureness index oscillates between 45 to $65 \%$ regardless the immunosuppressive scheme adopted or the interval time between the transplant and pregnancy. Still, there is some difficulty to set whether the loss of the graft function is due to the pregnancy or to factors attributed at the time of transplant. Study performed on pregnancy case-control after renal transplant did not reveal any influence on the pregnancy on the graft function. It was based on multi-centric reports documented in the National Transplantation Pregnancy Registry (N.T.P.R.). Concluding, the transplant rehabilitates the IRCT bearer to the pregnancy. On the other hand, the after renal transplant gestation does not represent a restricting factor to the maintenance of the allograft function.

Key words: pregnancy, post-renal transplantation, physiopathology, graft, kidney failure, chronic.

\section{REFERÊNCIAS}

1. MURRAY, J.E., REID, D. E., HARRISON, J.H., MERRILL, J.P. Successful pregnancies after human renal transplantation. N Engl J Med., 1963, 269:341-3.

2. SABER, L.T., DUARTE, G., COSTA, J.A., COlOGNA, A.J., GARCiA, T.M., FERRAZ, A.S. Pregnancy and kidney transplantation: experience in a developing country. Am J Kidney Dis., 1995, 25(3):465-70.

3. CARARACH, V., MONLEON, F .J. Pregnancy after renal transplantation: 25 years experience in Spain. British Journal of Obstetrics and Gynaecology, 1993; 100(2): $122-5$.

4. ARMENTI V.T., AHLSWEDE, K.M., AHLSWEDE, B.A., CATER, J.R., JARRELL, B.E., MORTIZ, M.J., ET AL. Variables affecting birth weight and graft survival in 197 pregnancies in cyclosporine - treated female kidney transplant recipients. Transplantation, 1995; 59(4): 476-9.

5. DAVISON, J.M., HYTTEN, F.E. Glomerular filtration during and after pregnancy. J Obstet Gyneacol Br Commonw, 1974; 81(8):588-95

6. ATHERTON, J.C., GREEN, R. Renal tubular function in the gravid rat. Baillier's Clin Gyneacol, 1994; 8:265-85

7. BOIM, M.A.; SCHOR, N. Trato urinário na gravidez: aspectos funcionais. In: SCHOR N.; SROUGI, M. Nefrologia, urologia, clínica. São Paulo, Sarvier, 1998; 181-5.

8. BAYLIS, C., RENNKE, H.G. Renal hemodynamics and glomerular morphology in repetitively pregnant aging rats. Kidney Int, 1985; 28(2):140-5.

9. DAVISON, J. M. Pregnancy in renal allograft recipients: prognosis and management Baillieres Clin Obstet Gynaecol, 1987; 1(4):1027-45.

10. DAVISON, J.M. Pregnancy in renal allograft recipients: problems, prognosis and practicalities. Baillieres Clin Obstet Gynaecol, 1994; 8(2):501-25.

11. COX. M.S., CUNNINGHAM, F.G. Blood volume change during pregnancy. Society of Perinatal Obstetric, Lousiana, 1989.

12. VENUTO, R.C., DONKER, A.J. Prostaglandin E2, plasma renin activity, and renal function throughout rabbit pregnancy. J Lab Clin Med, 1982, 99(2):239-46.

13. ELKARIB, A. O., GARLAND, H. O., GREEN, R. Acute and chronic affects of progesterone and prolactin on renal function in the rat. J Physiol, 1983; 337:389-400.

14. WALKER, J., GARLAND, H. O. Single nephron function during prolactin-induced pseudo pregnancy in the rat. J Endocrinol, 1985, 107(1):127-31,

15. GREEN, R., HATTON, T. M. Renal tubular function in gestation. Am J Kidney Dis, 1987, 9(4):265-9.

16. SLADEK, S.M., MAGNESS, R.R., CONRAD, K.P. Nitric oxide and pregnancy. Am J Physiol, 1997, 272(2 Pt2):441-63

17. CONRAD, K.P., COLPOYS, M.C. Evidence against the hypothesis that prostaglandins are the vasodepressor agents of pregnancy. Serial studies in chronically instrumented, conscious rats. J Clin Invest, 1986; 77(1):236-45

18. DANIELSON, L.A., CONRAD, K.P. Prostagladins maintain renal vasodilation and hyperfiltration during chronic nitric oxide synthase blockade in conscious pregnant rats. Circ Res, 1996; 79(6):1161-6.

19. CONRAD, K.P., BRINCK-JOHNSEN, T., ADLER, R.A. Evidence that chronic hyperprolactinemia increases renal hemodinamics. Clin. Res, 1986; 34:695-8.

20. ATALLAH, N.A. I. R.A. na gestação. In SHOR, N.; BOIN, M.A.; PAVÃO DOS SANTOS, O.F. (Ed.). Insuficiência renal aguda: fisiologia, clínica e tratamento. São Paulo, Sarvier, 1997; 223-6.

21. EROGLU, G., BETZ, G., TORREGANO, C. Impact of histocompatibility antigens on pregnancy outcome. Am. J. Obstet. Gynecol, 1992; 166(5):1364-9.

22. GAUGHAN, W.J., MORITZ, M.J., RADOMSKI, J. S., Burke, J.F. Jr., ARMENTI, V. T. National Transplantation Pregnancy Registry: report on outcomes in cyclosporinetreated female kidney transplant recipients with an interval from transplant to pregnancy of greater than five years. American Journal of kidney Diseases, 1996, 28(2):266-9.

23. SURANYI, M. G., HALL, B.M. Current status of renal transplantation. West J Med, 1990, 152(6):687-96.

24. FISCHER, T. H., SCHOBEL, H., BARENBROCK, M.. Specific immune tolerance during pregnancy after renal transplantation. European Journal of Obstetrics Gynecology Reproductive Biology, 1996, 70(2):217-9.

25. POLLACK, M. S., SHORT, H. D. 3rd., Young, J.B., Piwinski, S.E., Callaway, C., Debakey, M.E. Graft stability in a heart transplant recipient whose immunosuppressive therapy was discontinued for 8 months. Transplantation, 1988, 45(1):242-3.

26. STURGISS, S. N., DAVISON, J. M. Perinatal outcome in renal allograft recipients prognostic significance of hypertension and renal function before and during pregnancy. Obstet Gynecology, 1991, 78(4):573-7. 
27. DAVISON, J.M.: Dialysis, transplantation and pregnancy. Am. J. Kidney Dis, 1991; 17(2):127-31.

28. HADI, H.A: Pregnancy in renal transplant recipient: a review. Obster Gynecol Surg, 1986, 41(5):264-71.

29. ThOMAS, A. G., BURROWS, L., KNIGHT, R., PANICO, M., LAPINSKI, R., LOCKWOOD, C. J. The effect of pregnancy on cyclosporine levels in renal allograft patients. Obstet Gynecol, 1997, 90(6): 916-9.

30. SAlmela, K. T., KYllonen, L. E., HOlmberG, C., GRONHAGENRISKA, C. Impaired renal function after pregnancy in renal transplant recipients. Transplantation, 1993, 56(6):1372-5.

31. BIESENBACH, G., ZAZGORNIK, J., KAISER, W., STOGER, H., DERFLER, K., BALCKE, P., ET AL.. Cyclosporin requirement during pregnancy in renal transplant recipients. Nephrol Dial Transplant, 1989; 4(7):667-9.

32. CORRÊA, M. D. Parto Prematuro. In: REZENDE, J.; MONTENEGRO, C. A B Obstetrícia, 9 ed, Rio de Janeiro, Guanabara Koogan, 1998.

33. MUIRHEAD, N. SABHARWAL, A. R., RIEDER, M. J., LAZAROVITS, A.I., HOLLOMBY, D.J. The outcome of pregnancy following renal transplantation - the experience of a single center. Transplantation, 1992, 54(3):429-32.

34. StimsOn, W.H., STRACHAN, A. F., SHEPERD, A. Studies on the maternal immune response to placental antigens: absence of a blocking factor from blood of abortion - prone women. Br J Obstet Gynaecol, 1979, 86(1):41-5.

35. HALL, B.M. Tolerance and specific unresponsiveness in organ transplantation immunol. Aller. Clin. North, 1989, 1:61-77.

36. HALL, B.M. Transplantation tolerance: a 1988 perspective. Transplant Proc., 1989b, 21(1 Pt 1):816-9.
37. TAYLOR, C., FAULK, W. P. Prevention of recurrent abortion with leukocytes transfusions. Lancet, 1981, 2(8237):68-70.

38. SCOTT, J.R. Gynecologic and obstetric problems in renal allograft recipients. In Buchmawm, H.V., Shimidt, J., editors, Gynecologic urology, second edition, Philadelphia, W.B. Saunders, 1982, p. 547

39. FURMAN, B. WIZNITZER, A., HACKMON, R., GOHAR, J., MAZOR, M Multiple pregnancies in women after renal transplantation. Case report that rises management dilemma. European Journal of Obstetrics Gynecology and Reproductive Biology, 1999, 84(1):107-10.

40. LAU, R. J., SCOTT, J. R. Pregnancy following renal transplantation. Clin Obstet. Gynecol, 1985, 28(2):339-50.

41. EVANS, M.I., DOMMERGUeS, M., WAPNER, R.J., LYNCH, L., DUMEZ, Y., GOLDBERG, ET AL., JOHNSON, M.P., GOLBUS, M.S., et al. Efficacy of transabdominal multifetal pregnancy reduction: collaborative experience among the world's largest centers. Obstet Gynecol. 1993; 82(1):61-6.

42. SCIARRA, J.J., TOLEDO-PEREYRA, L.H., BENDEL, R.P., SIMMONS, R.L. Pregnancy following renal transplantation. Am J Obstet Gynecol., 1975, 123(4):411-25.

43. E.D.T.A. Successful pregnancies in women treated by dialysis and kidney transplantation. Report from the Registration Committee of the European Dialysis and Transplant Association (EDTA). Br J Obstet Gynaecol., 1980; 87(10):839-45

44. FINE, R.N. Pregnancy in renal allograft recipients. Am. J. Neprhol. 1982; 2(3):117-21.

45. RECKELHOFF, J.F. Age - related changes in renal hemodynamics in female rats: role of multiple pregnancies and NO. Am J Physiol., 1997, 272(6 Pt 2):R 1985-9. 